\title{
COMPLEX PROJECTIVE STRUCTURES WITH MAXIMAL NUMBER OF MÖBIUS TRANSFORMATIONS
}

\author{
GIANLUCA FARACO AND LORENZO RUFFONI
}

\begin{abstract}
We consider complex projective structures on Riemann surfaces and their groups of projective automorphisms. We show that the structures achieving the maximal possible number of projective automorphisms allowed by their genus are precisely the Fuchsian uniformizations of Hurwitz surfaces by hyperbolic metrics. More generally we show that Galois Belyı̆ curves are precisely those Riemann surfaces for which the Fuchsian uniformization is the unique complex projective structure invariant under the full group of biholomorphisms.
\end{abstract}

\section{Contents}

1. Introduction

2. Complex projective structures and their automorphisms

2.1. Basic definitions

2.2. Hurwitz projective structures

2.3. Relatively Hurwitz projective structures 6

3. An affine detour

3.1. Complex affine structures on tori $\quad 7$

3.2. Translation surfaces 9

4. Action of biholomorphisms on projective structures $\quad 10$

$\begin{array}{ll}\text { 4.1. Schwarzian parametrization } & 10\end{array}$

4.2. Criterion for projectiveness 11

5. Fuchsian uniformizations and Galois Belyı̆ curves 13

$\begin{array}{ll}\text { References } & 15\end{array}$

\section{INTRODUCTION}

Upgrading a smooth surface $S$ to a Riemann surface $X$ by introducing a complex structure turns it into a quite rigid object from several points of view. The prototypical phenomenon was discovered by Hurwitz in 1893, who proved in [Hur92] that, if the genus $g$ of the surface is at least 2, then the group of holomorphic automorphisms of $X$ is finite, the cardinality being bounded just in terms of the genus as \# Aut $(X) \leq 84(g-1)$. Riemann surfaces which attain this bound have

Date: November 14, 2019.

2010 Mathematics Subject Classification. 57M50,14H37.

Key words and phrases. Complex projective structures, Fuchsian groups, automorphisms, Galois Belyı̆ curves, quasiplatonic surfaces. 
been named Hurwitz surfaces and the finite groups arising as their groups of automorphisms have been named Hurwitz groups. A lot of research has then been done to study geometric and algebraic properties of these objects: see [Con10], [Con90], [Lev99] and the references therein.

It is natural to ask what happens if we further rigidify the geometry, for instance fixing a special coordinate covering for a Riemann surface (in the sense of [Gun67]), i.e. an open cover for which transition functions are not just local biholomorphisms, but belong to some more restricted group of transformations of the Riemann sphere $\mathbb{C P}^{1}$. Schlage-Puchta and Weitze-Schmithüsen in [SPWS17] consider the case of translation surfaces, for which transition functions are translations. They show that a result similar to Hurwitz's one holds, with an explicit bound being $4(g-1)$; they call Hurwitz translation surfaces the structures which achieve this bound, and characterise them as normal origamis, i.e. square-tiled surfaces which arise as Galois coverings of the standard torus $\mathbb{C} / \mathbb{Z}[i]$.

Here we consider the same questions for complex projective structures, i.e. geometric structures defined by an atlas of charts for which transitions are given by restrictions of global Möbius transformations of the Riemann sphere. Such a structure in particular induces a complex structure on the underlying surface, and indeed the interest in this kind of structures comes from classical uniformization theory (see [Gun67]) as well as from the study of linear ODEs (see [GKM00]). We show that the group of projective automorphisms of a complex projective structure can be as large as the group of holomorphic automorphisms of the underlying Riemann surface, and that the complex projective structures which attain this bound are precisely the ones arising from Fuchsian uniformizations of Hurwitz surfaces by hyperbolic metrics.

The classical approach to this kind of questions is to consider the quotient of a structure by its full group of automorphisms; however this does not work for projective structures, since the quotient might not exist as a projective structure. Our approach is to take a relative point of view: given a Riemann surface, we ask which are the complex projective structures on it for which the full group of holomorphic automorphisms acts by projective automorphisms; we call such structures relatively Hurwitz projective structures. For genus $g \geq 2$ Fuchsian uniformizations are always among them, but generically every structure has this property, just because a generic Riemann surface of genus $g \geq 3$ has no non-trivial automorphism. We identify the condition under which the Fuchsian uniformization is the only structure with such a property; our main result (see Theorem 5.4 below) can be stated as follows.

Theorem 1.1. Let $X$ be a Riemann surface of genus $g \geq 2$. Then the following are equivalent:

(i) $X$ is a Galois Belyॅ curve;

(ii) the Fuchsian uniformization of $X$ is the unique relatively Hurwitz projective structure in $\mathcal{P}(X)$.

An analogous statement holds in genus $g=1$ replacing "Fuchsian" by "Euclidean". The first condition in the above statement means that $X / \operatorname{Aut}(X)$ has genus zero and that $\pi: X \rightarrow X / \operatorname{Aut}(X)$ branches exactly over 3 points, so that $\pi$ is both a Belyı function on $X$ and a Galois cover; in particular these Riemann surfaces are examples of Bely $\breve{1}$ curves, i.e. algebraic curves which can be defined over the field of algebraic numbers $\overline{\mathbb{Q}}$. These curves are also known as quasiplatonic 
surfaces, and have been studied by many authors (see for instance [CV19], [JW16], [Pop72], [SPW06] and [Wol97]); they are known to be exactly those Riemann surfaces which have strictly more automorphisms than any proper deformations, or equivalently that can be uniformized by normal subgroups of hyperbolic triangle groups. A straightforward consequence of the theorem is that a complex projective structure has strictly more automorphisms than any proper deformation if and only if it is the Fuchsian uniformization of a Galois Belyı̆ curve; in particular we recover the aforementioned characterization of Hurwitz projective structures as Fuchsian uniformizations of Hurwitz Riemann surfaces (see Corollary 5.6 below).

As in the case of translation surfaces, we have therefore a very neat geometric description of the structures which attain the bound. Moreover in both cases these structures turn out to be integral points of their moduli spaces: normal origamis are among square-tiled surfaces, which are integral points in period coordinates (see [Zor06]), and Fuchsian uniformizations are among Fuchsian projective structures, which are integral points in Thurston's coordinates (see [Dum09]).

The paper is organised as follows: Section 2 contains the basics about complex projective structures and their automorphisms, and the definition of (relatively) Hurwitz projective structure; in Section 3 we describe the picture for genus 1 and for translation surfaces. In Section 4 we gather the technical lemmas about the Schwarzian parametrization of projective structures which are used in Section 5 to prove the main result; in particular a criterion is obtained for a biholomorphism $f \in \operatorname{Aut}(X)$ to act projectively on a given projective structure in terms of an affine action of $\operatorname{Aut}(X)$ on the space of holomorphic quadratic differentials $H^{0}\left(X, K^{2}\right)$.

Acknowledgements: We would like to thank Stefano Francaviglia for his encouragement in developing this project, Pascal Hubert for pointing us to useful references, and the referees for carefully reading this paper. The second author acknowledges support by INDAM-GNSAGA.

\section{Complex Projective Structures AND Their AUtomorphisms}

In this section we gather the needed preliminaries on complex projective structures that we will use in the sequel.

2.1. Basic definitions. Let $S$ be a closed, connected and orientable surface, let $\mathbb{C P}^{1}=\mathbb{C} \cup\{\infty\}$ be the Riemann sphere and let $\mathrm{PSL}_{2} \mathbb{C}$ be its group of holomorphic automorphisms acting by Möbius transformations

$$
\mathrm{PSL}_{2} \mathbb{C} \times \mathbb{C P}^{1} \rightarrow \mathbb{C P}^{1},\left(\begin{array}{ll}
a & b \\
c & d
\end{array}\right), z \mapsto \frac{a z+b}{c z+d}
$$

Definition 2.1. A complex projective structure $\sigma$ on $S$ is a maximal atlas of charts taking values in $\mathbb{C P}^{1}$ and such that transition functions are restrictions of elements in $\mathrm{PSL}_{2} \mathbb{C}$.

In the following we will also refer to such structures simply as projective structures. Given a projective structure $\sigma$, performing analytic continuation of local charts of $\sigma$ along paths in $S$ gives rise to an immersion dev $: \widetilde{S} \rightarrow \mathbb{C P}^{1}$, usually called a developing map for $\sigma$, well-defined up to post-composition by a Möbius transformation. We refer to [Dum09] for a detailed survey about complex projective structures. 
Remark 2.2. Since Möbius transformations are in particular holomorphic maps, a projective structure on $S$ always determines an underlying Riemann surface structure on $S$. Conversely, by the classical uniformization theory, any Riemann surface $X$ is of the form $U / \Gamma$ where $U$ is an open subset of $\mathbb{C P}^{1}$ and $\Gamma$ is a discrete subgroup of $\mathrm{PSL}_{2} \mathbb{C}$ acting freely and properly discontinuously on $U$; this endows $X$ with a complex projective structure, namely the one coming from the identification $X \cong U / \Gamma$. When $g=0$ there is only one possible choice $U=\mathbb{C P}^{1}$ and $\Gamma=1$. If $g=1$ then $U$ can be chosen to be the complex plane $\mathbb{C}$ and $\Gamma$ to be a discrete free abelian group of rank 2 acting by translations. When $g \geq 2$ it is possible to choose $U$ to be the upper-half plane $\mathcal{H}=\{z \in \mathbb{C} \mid \operatorname{Im}(z)>0\}$ and $\Gamma$ to be a Fuchsian group, i.e. a discrete subgroup of $\mathrm{PSL}_{2} \mathbb{R}$. Notice in particular that for a projective structure of this type any developing map is a diffeomorphism dev $: \widetilde{S} \rightarrow U$, and that it endows $X$ with a canonical Riemannian metric of constant sectional curvature $k=\operatorname{sign}(\chi(X)) \in\{1,0,-1\}$.

Definition 2.3. Let $X$ be a Riemann surface of genus $g$. If $\Gamma \subset \mathrm{PSL}_{2} \mathbb{C}$ is discrete, $U \subset \mathbb{C P}^{1}$ is an open connected domain on which $\Gamma$ acts freely and properly discontinuously with quotient $U / \Gamma$ diffeomorphic to $S$, then we say that the projective structure $\sigma=U / \Gamma$ is a uniformization of $X$. In particular if $g=1$ then we call the Euclidean uniformization of $X$ the complex projective structure coming from the flat Riemannian metric as in Remark 2.2, and if $g \geq 2$ then we call the Fuchsian uniformization of $X$ the complex projective structure coming from the hyperbolic Riemannian metric as in Remark 2.2.

Remark 2.4. Among all possible ways of realising a Riemann surface $X$ as a quotient $U / \Gamma$ as in Remark 2.2, Euclidean and Fuchsian uniformizations are special in that $U$ is simply connected and $\Gamma$ is isomorphic to the fundamental group $\pi_{1}(X)$; other uniformizations arise for instance from Schottky uniformization, where $U$ is the complement of a Cantor set and $\Gamma$ is a (non-abelian) free group. On the other hand, not every complex projective structure is of the form $U / \Gamma$ : for instance in [Mas69] Maskit has produced many examples of projective structures with surjective and non injective developing maps.

Now we turn to the study of maps between projective structures.

Definition 2.5. Let $\sigma_{1}, \sigma_{2}$ be projective structures on $S$ and let $f: \sigma_{1} \rightarrow \sigma_{2}$ be a diffeomorphism. We say that $f$ is projective if its restrictions to local projective charts are given by elements in $\mathrm{PSL}_{2} \mathbb{C}$. We say that $\sigma_{1}$ and $\sigma_{2}$ are isomorphic if there exists a projective diffeomorphism between them.

Definition 2.6. Let $\sigma$ be a projective structure. We define the group of projective automorphisms of $\sigma$ to be $\operatorname{Möb}(\sigma)=\{f \in \operatorname{Diff}(S) \mid f$ is projective for $\sigma\}$.

Up to isomorphism there is a unique complex projective structure $\sigma$ on a surface of genus 0 , given by its realisation as $\mathbb{C P}^{1}$; of course $\operatorname{Möb}(\sigma)=\operatorname{PSL}_{2} \mathbb{C}$. The following straightforward observations will be useful in the following.

Lemma 2.7. Let $\sigma$ be a projective structure on $S$ and dev $: \widetilde{S} \rightarrow \mathbb{C P}^{1}$ be a developing map for $\sigma$. Then $f \in \operatorname{Diff}(S)$ is projective if and only if there exists $g \in \mathrm{PSL}_{2} \mathbb{C}$ such that dev $\circ \widetilde{f}=g \circ$ dev, for some lift $\widetilde{f}$ of $f$ to $\widetilde{S}$.

Let us denote by $\operatorname{Aut}(X)$ the group of holomorphic automorphisms of a Riemann surface $X$. The following is immediate. 
Lemma 2.8. Let $\sigma$ be a projective structure on $S$ and let $f \in \operatorname{Möb}(\sigma)$. If $X$ is the underlying Riemann surface, then $f \in \operatorname{Aut}(X)$.

2.2. Hurwitz projective structures. From now on, unless otherwise specified, we assume that the surface $S$ has genus $g \geq 2$. Let $\sigma$ be a projective structure on $S$ and let $X$ be the underlying Riemann surface. By the classical Hurwitz Theorem the group $\operatorname{Aut}(X)$ of holomorphic automorphism of $X$ is a finite group of cardinality \#Aut $(X) \leq 84(g-1)$ (see [FK92, p. V.1.3]). A Hurwitz surface is classically defined to be a Riemann surface of genus $g$ whose automorphism group attains this bound. By the above Lemma 2.8 the same bound holds for the group Möb $(\sigma)$ of $\sigma$, and one can ask if it can be sharpened or not. A straightforward approach is to pick a Hurwitz Riemann surface $X$ and look for projective structures for which holomorphic automorphisms are also projective. This might be hard to check in general, but the collection of uniformizations of $X$ (see

Definition

2.3) provides a playground where we can perform concrete computations.

Lemma 2.9. Let $X$ be a Riemann surface and $\sigma=U / \Gamma$ a uniformization of $X$. Suppose that every $f \in \operatorname{Aut}(X)$ lifts to some $\tilde{f} \in \operatorname{Aut}(U)$ and that $\tilde{f}$ is the restriction of a Möbius transformation. Then $\operatorname{Möb}(\sigma)=\operatorname{Aut}(X)$.

Proof. By classical covering space theory, lifts of automorphisms of $X$ are exactly given by automorphisms of $U$ which normalise $\Gamma$ (both in the holomorphic and in the projective setting); in other words the normalizer $N_{\operatorname{Aut}(U)}(\Gamma)$ of $\Gamma$ in $\operatorname{Aut}(U)$ acts by biholomorphisms on $X$, the normalizer $N_{\operatorname{Möb}(U)}(\Gamma)$ of $\Gamma$ in $\operatorname{Möb}(U)$ acts by projective diffeomorphisms on $\sigma$ and both actions factor exactly through the standard action of $\Gamma$ by deck transformations of the covering $U \rightarrow U / \Gamma$; so we get injective maps $N_{\mathrm{Aut}(U)}(\Gamma) / \Gamma \rightarrow \operatorname{Aut}(X)$ and $N_{\operatorname{Möb}(U)}(\Gamma) / \Gamma \rightarrow \operatorname{Möb}(\sigma)$. The hypothesis imply that $N_{\operatorname{Aut}(U)}(\Gamma) / \Gamma \rightarrow \operatorname{Aut}(X)$ is surjective and that $N_{\operatorname{Aut}(U)}(\Gamma) / \Gamma=$ $N_{\operatorname{Möb}(U)}(\Gamma) / \Gamma$. In particular we obtain an injective map $\operatorname{Aut}(X) \rightarrow \operatorname{Möb}(\sigma)$. Since by Lemma 2.8 we always have $\operatorname{Möb}(\sigma) \subseteq \operatorname{Aut}(X)$ and both groups are finite, we get the result.

We can use the previous lemma to obtain that in general the Hurwitz bound can not be improved.

Lemma 2.10. Let $X$ be a Hurwitz surface and $\sigma$ its Fuchsian uniformization. Then $\operatorname{Möb}(\sigma)=\operatorname{Aut}(X)$; in particular \#Möb $(\sigma)=84(g-1)$.

Proof. By definition of Fuchsian uniformization, $\sigma$ is of the form $\mathcal{H} / \Gamma$, where $\mathcal{H}$ is the upper-half plane and $\Gamma \subset \mathrm{PSL}_{2} \mathbb{R}$ is a Fuchsian group. In particular $\mathcal{H}$ is simply connected and it is well-known that its biholomorphisms are exactly given by Möbius transformations with real coefficients, i.e. $\operatorname{Aut}(\mathcal{H})=\operatorname{Möb}(\mathcal{H})=\mathrm{PSL}_{2} \mathbb{R}$; therefore we can just apply Lemma 2.9 .

We give therefore the following definition, by analogy with the classical case.

Definition 2.11. Let $\sigma$ be a projective structure on $S$. We say that $\sigma$ is a Hurwitz projective structure if $\# \operatorname{Möb}(\sigma)=84(g-1)$. 
Lemma 2.10 can then be restated by saying that Fuchsian uniformizations of Hurwitz surfaces are Hurwitz projective structures. Conversely one can ask if projective structures with a maximal number of projective automorphisms are necessarily given by hyperbolic metrics on Hurwitz surfaces. The answer turns out to be positive, and we will recover this result as consequence of a more general statement below (see Corollary 5.6).

Remark 2.12. The properties of Fuchsian uniformizations which we have used in the proof of Lemma 2.10 to apply Lemma 2.9 are the following: the domain $U$ is simply connected and $\operatorname{Aut}(U)=\operatorname{Möb}(U)$. By [Min79] such properties are known to characterise Fuchsian uniformizations. The same kind of arguments might fail for other uniformizations. For instance if $U$ is an open quadrant then it is simply connected, but not every biholomorphism is the restriction of a Möbius transformation. On the other hand if $U$ is a Schottky domain then it is not simply connected, but every biholomorphism is indeed projective (by [AS60, IV.2D,IV.19F]).

Remark 2.13. The study of geometric automorphisms of surfaces is usually done by taking the quotient by the action of the automorphism group, and studying the geometry and combinatorics of the resulting quotient map. In general this action may have fixed points, so that the quotient is endowed with an orbifold geometric structure. In the case of Riemann surfaces it turns out that the orbifold charts can actually be uniformized in such a way that the quotient carries a honest complex structure with respect to which the projection is holomorphic (see [Mir95, Theorem III.3.4]). Translation surfaces are more rigid, but the key observation (see [SPWS17]) is that fixed points of a translation can appear only at cone points of the structure, so that the effect of taking the quotient is absorbed in the reduction of the total curvature, and also in this case the quotient is endowed with a honest translation structure. In both cases this is quite useful in taming the behaviour of the quotient map (e.g. its degree). On the other hand, for complex projective structures it is in general not possible to reuniformize the orbifold charts in such a way that the quotient supports a honest projective structure with respect to which the quotient map is projective. Consider for instance a closed surface of genus $g \geq 2$ endowed with a hyperelliptic hyperbolic structure (seen as a projective structure here); the structure induced on the quotient is a hyperbolic cone structure on a genus 0 surface, which is of course not the standard projective structure on $\mathbb{C P}^{1}$. We will bypass this issue by looking at the action of the automorphism group on the space of holomorphic quadratic differentials, which is classically known to provide a parametrization of the space of projective structures (see 4.5 below). The previous comments translate into this setting by saying that a holomorphic quadratic differential descends to a meromorphic, but in general not holomorphic, differential on the quotient (see Remark 4.6 below).

2.3. Relatively Hurwitz projective structures. Our approach to the problem will be to consider it in a relative way: any projective structure has an underlying complex one, and by Lemma 2.8 any projective automorphism is in particular holomorphic for it; a natural question is to ask when every holomorphic automorphism is indeed projective, which motivates the following definition.

Definition 2.14. Let $\sigma$ be a projective structure on $S$ and $X$ the underlying Riemann surface. We say that $\sigma$ is a relatively Hurwitz projective structure if $\operatorname{Möb}(\sigma)=\operatorname{Aut}(X)$. 
Example 2.15. A generic Riemann surface $X$ of genus $g \geq 3$ has no non-trivial automorphisms, therefore any projective structure on $X$ is a relatively Hurwitz projective structure.

Example 2.16. On the other end of the spectrum, let $X$ be a Hurwitz surface. By Lemma 2.10 the Fuchsian uniformization $\sigma$ of $X$ is a relatively Hurwitz projective structure: any holomorphic automorphism is indeed an isometry for the uniformizing hyperbolic metric, and those are all projective.

More generally, we can apply Lemma 2.9 as in Lemma 2.10 to obtain the following.

Lemma 2.17. Let $X$ be any Riemann surface and $\sigma$ its Fuchsian uniformization. Then $\operatorname{Möb}(\sigma)=\operatorname{Aut}(X)$; in particular $\sigma$ is a relatively Hurwitz projective structure.

Example 2.18. Non-trivial examples of relatively and non-relatively Hurwitz projective structures will be given below in Example 4.11 on hyperelliptic Riemann surfaces of genus $g \geq 3$ whose automorphism group is generated by the hyperelliptic involution.

The following question turns out to hide rich geometric phenomena: when is it true that the Fuchsian uniformization of $X$ is the unique relatively Hurwitz projective structure? As a warm-up, we will first consider the analogous problem in other settings, namely translation surfaces and elliptic curves.

\section{An AFFine DETOUR}

In this section we gather some results available in the literature in order to describe the geometric features enjoyed by geometric structures with maximal group of automorphisms in the setting of affine geometry.

3.1. Complex affine structures on tori. In this section (and only in this section) we consider the situation in which $S$ has genus $g=1$. In this case complex projective structures are actually complex affine structures (see [Gun67]), i.e. the local charts take values in a fixed affine patch $\mathbb{C} \subset \mathbb{C P}^{1}$ and the changes of coordinates are restrictions of complex affine transformations $g \in \operatorname{Aff}_{1}(\mathbb{C})=\{z \mapsto a z+b \mid a \in$ $\left.\mathbb{C}^{*}, b \in \mathbb{C}\right\}$. These geometric structures are conveniently described as affine deformations of the Euclidean uniformizations (see [Gun67] or [LMP09] for more details). More precisely let $\tau \in \mathcal{H}^{+} / \mathrm{SL}_{2} \mathbb{Z}=\{\operatorname{Im}(z)>0\} / \mathrm{SL}_{2} \mathbb{Z}, \Lambda_{\tau}=\operatorname{span}_{\mathbb{Z}}\{1, \tau\}$ and $X_{\tau}=\mathbb{C} / \Lambda_{\tau}$. The complex affine structures on $X_{\tau}$ are parametrized by $c \in \mathbb{C}$ : for $c=0$ we have the Euclidean uniformization $\sigma_{E}$ of $X_{\tau}$ (see Remark 2.2); for $c \in \mathbb{C}^{*}$ we get a non-Riemannian affine structure $\sigma_{c}$ on $X_{\tau}$ defined by a developing map $\operatorname{dev}_{c}: \mathbb{C} \rightarrow \mathbb{C}, z \mapsto e^{c z}$. The group of automorphisms $\operatorname{Aut}\left(X_{\tau}\right)$ of the torus $X_{\tau}$ is the semidirect product $\operatorname{Aut}\left(X_{\tau}\right)=X_{\tau} \rtimes \operatorname{Aut}^{0}\left(X_{\tau}\right)$, where $X_{\tau}$ acts on itself by translations $T_{p}(z)=z+p$ for $p \in X_{\tau}$ and $\operatorname{Aut}^{0}\left(X_{\tau}\right)$ is a finite group which generically consists only of the hyperelliptic involution $J(z)=-z$, and also contain a complex multiplication $R_{\tau}(z)=\tau z$, when $\tau=e^{i \frac{2 \pi}{3}}$ or $\tau=e^{i \frac{\pi}{2}}$. A straightforward computation shows that the translation part always acts by complex affine transformations: this is clear for the Euclidean uniformization, and for the other structures we just observe that a translation acts as $\operatorname{dev}_{c}\left(T_{p}(z)\right)=\operatorname{dev}_{c}(z+p)=e^{c(z+p)}=e^{c p} \operatorname{dev}_{c}(z)$, i.e. as a complex dilation, and use Lemma 2.7. 
Definition 3.1. Let $X$ be a Riemann surface of genus 1 . We denote by $\mathcal{A}(X)$ the space of complex affine structures on $X$. If $\sigma \in \mathcal{A}(X)$, then we denote by $\operatorname{Aff}_{1}(\sigma)$ the group of affine automorphisms, i.e. the ones that are given by elements in $\operatorname{Aff}_{1}(\mathbb{C})$ in affine charts for $\sigma$, and by $\operatorname{Aff}_{1}^{0}(\sigma)$ the reduced group obtained by quotienting out the translations, so that $\operatorname{Aff}_{1}(\sigma)=X_{\tau} \rtimes \operatorname{Aff}_{1}^{0}(\sigma)$.

Notice that in general we only have $\operatorname{Aff}_{1}^{0}(\sigma) \subseteq \operatorname{Aut}^{0}(X)$. In particular $\operatorname{Aff}_{1}^{0}(\sigma)$ has at most order 6 , which is the bound for the cardinality of the reduced automorphism group $\operatorname{Aut}^{0}(X)$ of a Riemann surface of genus 1 .

Example 3.2. A direct computation shows that actually each automorphism of $X$ is a complex affine transformation (indeed a Euclidean isometry) with respect to its Euclidean uniformization $\sigma_{E}$, i.e. $\operatorname{Aut}(X)=\operatorname{Aff}_{1}\left(\sigma_{E}\right)$. In particular $\operatorname{Aff}_{1}^{0}\left(\sigma_{E}\right)=\operatorname{Aut}^{0}(X)$; therefore the Euclidean uniformization of the complex torus $X_{e^{i \frac{2 \pi}{3}}}$ achieves the maximum possible cardinality.

We are naturally led to give the following definitions.

Definition 3.3. Let $X$ be a Riemann surface of genus 1 and $\sigma \in \mathcal{A}(X)$; we say $\sigma$ is a Hurwitz affine structure if \# $\operatorname{Aff}_{1}^{0}(\sigma)=6$, and that it is a relatively Hurwitz affine structure if $\operatorname{Aff}_{1}^{0}(\sigma)=\operatorname{Aut}^{0}(X)$.

We then have the following.

Proposition 3.4. The unique Hurwitz affine structure is the Euclidean uniformization of $X_{e^{i \frac{2 \pi}{3}}}$. For a Riemann surface $X$ of genus 1 the unique relatively Hurwitz affine structure in $\mathcal{A}(X)$ is its Euclidean uniformization.

Proof. The first statement follows from the above discussion and the fact that $X_{e^{i \frac{2 \pi}{3}}}$ is the only torus with $\operatorname{Aut}^{0}\left(X_{e^{i \frac{2 \pi}{3}}}\right)=6$. For the second statement fix $X$, let $J \in \operatorname{Aut}(X)$ be the hyperelliptic involution and assume $\sigma$ is not the Euclidean uniformization. Then we just compute that $J$ acts as $\operatorname{dev}_{c}(J(z))=\operatorname{dev}_{c}(-z)=$ $e^{-c z}=\frac{1}{\operatorname{dev}_{c}(z)}$, i.e. as an inversion, which is not affine.

Looking at affine structures from a projective point of view, we see that the Euclidean uniformization of $X_{e^{i \frac{2 \pi}{3}}}$ is a Hurwitz projective structure (by Example 3.2). The same ideas as above also prove the following.

Proposition 3.5. Let $X_{\tau}$ be a Riemann surface of genus 1. Then the following are equivalent

(i) $\tau=e^{i \frac{2 \pi}{3}}$ or $\tau=e^{i \frac{\pi}{2}}$

(ii) the Euclidean uniformization of $X_{\tau}$ is the unique relatively Hurwitz projective structure in $\mathcal{A}\left(X_{\tau}\right)$.

Proof. From the previous proof of Proposition 3.4 we know that the hyperelliptic involution does not act affinely, but at least it always acts projectively. But if we consider surfaces which admit complex multiplication, we see that $R_{\tau} \in \operatorname{Aut}\left(X_{\tau}\right)$ acts on a structure which is not the Euclidean one as $\operatorname{dev}_{c}\left(R_{\tau}(z)\right)=\operatorname{dev}_{c}(\tau z)=$ $e^{\tau c z}=\operatorname{dev}_{c}(z)^{\tau}$, i.e. not projectively.

We see therefore that requiring the Euclidean uniformization to be the unique relatively Hurwitz projective structure picks out special points in the moduli space of genus 1 Riemann surfaces, namely those having extra automorphisms, or equivalently, those corresponding to regular lattices. In the following sections we are going 
to extend this result to higher genus surfaces, replacing Euclidean uniformization by Fuchsian uniformization.

3.2. Translation surfaces. We now turn to consider translations surfaces. These can be defined either as couples $(X, \omega)$ where $X$ is a Riemann surface and $\omega \in$ $H^{0}(X, K), \omega \neq 0$, or as complex projective structures with change of coordinates given by translations and with branch points (corresponding to the zeroes of $\omega$ ); see [Zor06] for more details.

The problem of counting translation automorphisms of a translation surface, and of describing the geometric features of structures maximizing this number, has been considered in [SPWS17], where the following definition is introduced.

Definition 3.6. Let $(X, \omega)$ be a translation surface. Denote by $\operatorname{Trans}(X, \omega)$ the group of automorphisms of $X$ which are given by translations in local charts for the translation structure.

Then the following result is obtained in [SPWS17].

Theorem 3.7 (Schlage-Puchta, Weitze-Schmithüsen [SPWS17]). Let $(X, \omega)$ be a translation surface. Then \#Trans $(X, \omega) \leq 4(g-1)$. Moreover \# Trans $(X, \omega)=$ $4(g-1)$ (i.e. $(X, \omega)$ is a Hurwitz translation surface) if and only if $(X, \omega)$ is a normal origami.

Recall that origamis (also known as square-tiled surfaces) are a very special type of translation surfaces; they can be defined as those $(X, \omega)$ arising as a covering of the standard torus $\mathbb{C} / \mathbb{Z}[i]$ branched exactly at one point, and the normal ones are those for which this covering is normal. The moduli space of translation surfaces carries a natural action by $\mathrm{GL}_{2} \mathbb{R}$ : given a translation structure $(X, \omega)$ and a matrix $A \in \mathrm{GL}_{2} \mathbb{R}$ we can obtain a new translation structure $A .(X, \omega)$ by just multiplying by $A$ every chart from a translation atlas for $(X, \omega)$; in general this will change the underlying complex structure unless $A \in \mathrm{GL}_{1} \mathbb{C}<\mathrm{GL}_{2} \mathbb{R}$ (see [Zor06] for more details). Notice however that $(X, \omega)$ and $A .(X, \omega)$ have the same translation automorphisms: if a diffeomorphism $f \in \operatorname{Diff}(S)$ is expressed as $f(z)=z+v$ in local charts for $(X, \omega)$ then it is given by $f(w)=w+A v$ in the corresponding local charts for $A .(X, \omega)$. Therefore the above statement should be considered up to this $\mathrm{GL}_{2} \mathbb{R}$ action: Hurwitz translation surfaces are those whose $\mathrm{GL}_{2} \mathbb{R}$-orbit contains a normal cover of the standard torus $\mathbb{C} / \mathbb{Z}[i]$ branched over a single point. For the sake of completeness, we now discuss what would happen in the context of translation surfaces when taking a relative point of view analogous to the one we are adopting here.

Definition 3.8. A translation surface $(X, \omega)$ is a relatively Hurwitz translation surface if $\operatorname{Aut}(X)=\operatorname{Trans}(X, \omega)$.

If looking for Hurwitz objects has led to the consideration of Fuchsian/Euclidean uniformizations in the previous setting (see Lemma 2.10 and Example 3.2), looking at Hurwitz translation surfaces suggests to look for the property of being a normal origami. One could ask, as before, if there is an interesting relation between this geometric feature and the relative Hurwitz condition. The main difference with respect to the previous setting is that, by the above bound in Theorem 3.7, relatively Hurwitz translation structures simply do not exist on Riemann surfaces which have too many automorphisms, and normal origamis do not exist on Riemann surfaces 
which do not have enough. On the other hand, if \#Aut $(X)=4(g-1)$, then it is straightforward to see that relatively Hurwitz translation surfaces on $X$ are precisely given by the normal origamis on it (up to $\mathrm{GL}_{2} \mathbb{R}$-action), just by checking cardinalities and applying Theorem 3.7.

Remark 3.9. Given a Riemann surface $X$, relatively Hurwitz translation surfaces $(X, \omega)$ are precisely defined by non-zero fixed points for the natural linear action of Aut $(X)$ on $H^{0}(X, K)$, because translation automorphisms are exactly those which preserve the abelian differential. By [FK92, Corollary V.2.2] the invariant differentials form a subspace of dimension equal to the genus of the quotient $X / \operatorname{Aut}(X)$. When \# Aut $(X)=4(g-1)$, this quotient can have genus either 0 or 1; a relatively Hurwitz translation structure exists on $X$ precisely when it is 1 and $X \rightarrow X / \operatorname{Aut}(X)$ is ramified over only one point. In particular if such a structure $(X, \omega)$ exists over $X$, then there is precisely a 1-dimensional family of them: this is precisely the orbit of $(X, \omega)$ under $\mathrm{GL}_{1} \mathbb{C}<\mathrm{GL}_{2} \mathbb{R}$. Concretely, these structures are obtained by deforming it only by rotations and dilatations; this is the same as pulling back the differential $\lambda d z$ on the quotient torus via the covering $X \rightarrow X / \operatorname{Aut}(X)$, varying the parameter $\lambda \in \mathbb{C}^{*}$. A necessary condition for a normal origami to exist on a surface of genus $g$ was identified by group-theoretic techniques in [SPWS17], namely $g-1$ must be divisible by 2 or 3 .

\section{ACtion of Biholomorphisms on projective STRUCTURES}

In this section we review the classical Schwarzian parametrization for complex projective structures with a fixed underlying complex structure (in the sense of Remark 2.2), in order to fix terminology and notation.

Definition 4.1. Let $X$ be a Riemann surface. We denote by $\mathcal{P}(X)$ the set of projective structures whose underlying complex structure is $X$.

We will prove a criterion for a biholomorphism to be projective for a given projective structure in terms of its action on the space of holomorphic quadratic differentials.

4.1. Schwarzian parametrization. The classical parametrization of $\mathcal{P}(X)$ by holomorphic quadratic differentials (see [Dum09], [Gun67]) is achieved by means of the following differential operator.

Definition 4.2. Let $\Omega \subset \mathbb{C}$ be an open domain and $f: \Omega \rightarrow \mathbb{C}$ be a holomorphic and locally injective function. The Schwarzian derivative of $f$ is defined to be

$$
\mathcal{S}(f)=\left(\frac{f^{\prime \prime}}{f^{\prime}}\right)^{\prime}-\frac{1}{2}\left(\frac{f^{\prime \prime}}{f^{\prime}}\right)^{2}
$$

The basic and well-known properties of this operator are the following.

Lemma 4.3. Let $\Omega \subset \mathbb{C}$ be open and $f, g: \Omega \rightarrow \mathbb{C}$ be holomorphic functions such that $g(\Omega) \subset \Omega$. Then $\mathcal{S}(f \circ g)=(\mathcal{S}(f) \circ g) \cdot g^{\prime 2}+\mathcal{S}(g)$.

Lemma 4.4. Let $\Omega \subset \mathbb{C}$ be open and $f: \Omega \rightarrow \mathbb{C}$ be holomorphic function. Then $\mathcal{S}(f)=0$ if and only if $f$ is the restriction of a Möbius transformation.

A direct consequence is the following: given a projective structure $\sigma_{0}$ over a Riemann surface $X$ and a holomorphic map $f: \widetilde{X} \rightarrow \mathbb{C P}^{1}$ we can lift the projective 
structure from $X$ to $\widetilde{X}$ and we can compute the Schwarzian derivative of $f$ in local projective coordinates on $\widetilde{X}$. By the above formulae, we get a collection of locally defined functions on $\widetilde{X}$, which pack together to define a quadratic differential on $\widetilde{X}$; this is actually invariant under covering transformations, and thus descends to a well-defined quadratic differential on $X$, which we denote $\mathcal{S}_{\sigma_{0}}(f)$. In particular we can give the following definition.

Definition 4.5. Let $\sigma_{0}$ and $\sigma$ be projective structures on $X$. The Schwarzian derivative of $\sigma$ with respect to $\sigma_{0}$ is the holomorphic quadratic differential on $X$ given by $\mathcal{S}_{\sigma_{0}}(\sigma)=\mathcal{S}_{\sigma_{0}}($ dev $)$, where dev $: \widetilde{X} \rightarrow \mathbb{C P}^{1}$ is any developing map for $\sigma$.

It is a classical result (see [Gun67]) that, for any fixed $\sigma_{0} \in \mathcal{P}(X)$ the map

$$
\mathcal{P}(X) \rightarrow H^{0}\left(X, K^{2}\right), \sigma \mapsto \mathcal{S}_{\sigma_{0}}(\sigma)
$$

is a bijection with the vector space of holomorphic quadratic differentials on $X$, whose dimension is $3 g-3$ by Riemann-Roch. Of course the zero differential corresponds to the chosen projective structure $\sigma_{0}$; thus the set $\mathcal{P}(X)$ is naturally endowed with the structure of complex affine space.

Remark 4.6. From this point of view it is easier to make the observations contained in Remark 2.13 clearer. Indeed fix some background $\sigma_{0} \in \mathcal{P}(X)$ and pick $\sigma \in \mathcal{P}(X)$; if it were possible to consider the quotient $\pi: \sigma \rightarrow \sigma / \operatorname{Möb}(\sigma)$ in the category of projective structures, then in particular $\pi$ should send the holomorphic quadratic differential $q=\mathcal{S}_{\sigma_{0}}(\sigma)$ on $X$ to a holomorphic quadratic differential on the Riemann surface $X / \operatorname{Möb}(\sigma)$ corresponding to $\sigma / \operatorname{Möb}(\sigma)$. For this to happen we need $\pi$ to branch in a controlled way with respect to the zero divisor of $q$ : if $x \in X$ is a point at which the stabilizer of the action of $\operatorname{Möb}(\sigma)$ has order $m \geq 1$ and at which $q$ has a zero of order $s \geq 0$, then the induced (meromorphic) quadratic differential on $X / \operatorname{Möb}(\sigma)$ will have order $\frac{s-2(m-1)}{m}$, so that it is holomorphic when $s \geq 2(m-1)$, but has a genuine pole otherwise. For a concrete example consider the case in which $\operatorname{Möb}(\sigma)$ is large enough to guarantee that $X / \operatorname{Möb}(\sigma)$ has genus 0 (e.g. the Fuchsian uniformization for a Hurwitz surface or for a hyperelliptic one): in this case the quotient admits no holomorphic quadratic differential whatsoever.

4.2. Criterion for projectiveness. We are now going to consider the action of the biholomorphism group $\operatorname{Aut}(X)$ of $X$ on the space $\mathcal{P}(X)$ of projective structures on $X$. If $\sigma \in \mathcal{P}(X)$ is defined by a developing map dev $: \widetilde{X} \rightarrow \mathbb{C P}^{1}$ and $F \in \operatorname{Aut}(X)$, then $F . \sigma$ is defined to be the projective structure with dev $\circ \widetilde{F}^{-1}: \widetilde{X} \rightarrow \mathbb{C P}^{1}$ as a developing map for some lift of $F$ to the universal cover. Since $F$ is holomorphic on $X$, we have that F. $\sigma$ is again inside $\mathcal{P}(X)$.

Now let us fix a projective structure $\sigma_{0} \in \mathcal{P}(X)$. By the above discussion we get an identification $\mathcal{P}(X) \cong H^{0}\left(X, K^{2}\right)$ and we can look at the induced action on the space of holomorphic differentials, which we denote by $(F, q) \mapsto F . q$. Let us denote by $F^{*} q=q \circ F^{-1}$ the usual action of the automorphism group on the space of differentials by pullback. Then a direct computation using the properties of the Schwarzian derivative in Lemma 4.3 and Lemma 4.4 shows the following.

Lemma 4.7. Let $F \in \operatorname{Aut}(X)$. If $q=\mathcal{S}_{\sigma_{0}}(\sigma)$ for some $\sigma \in \mathcal{P}(X)$ then $F . q=$ $F^{*} q+\mathcal{S}_{\sigma_{0}}\left(\widetilde{F}^{-1}\right)$.

Proof. By definition $\mathcal{S}_{\sigma_{0}}(\sigma)=\mathcal{S}_{\sigma_{0}}($ dev $)$ for some developing map and $\mathcal{S}_{\sigma_{0}}(F . \sigma)=$ $\mathcal{S}_{\sigma_{0}}\left(\operatorname{dev} \circ \widetilde{F}^{-1}\right)$. 
In other words $\operatorname{Aut}(X)$ acts affinely on $H^{0}\left(X, K^{2}\right)$, with linear part given by the classical action by pullback and the translation part accounts for the initial choice of the projective structure $\sigma_{0}$. This action can be used to obtain the following criterion.

Proposition 4.8. Let $F \in \operatorname{Aut}(X), \sigma \in \mathcal{P}(X)$ and $q=\mathcal{S}_{\sigma_{0}}(\sigma)$. Then $F \in \operatorname{Möb}(\sigma)$ if and only if $F . q=q$.

Proof. Let dev be a developing map for $\sigma$; then $q=\mathcal{S}_{\sigma_{0}}(d e v)$. If $F \in \operatorname{Möb}(\sigma)$ then by Lemma 2.7 we have dev $\circ \widetilde{F}^{-1}=g \circ$ dev for some $g \in \mathrm{PSL}_{2} \mathbb{C}$. Therefore $F . q=\mathcal{S}_{\sigma_{0}}\left(\right.$ dev $\left.\circ \widetilde{F}^{-1}\right)=\mathcal{S}_{\sigma_{0}}(g \circ$ dev $)=\mathcal{S}_{\sigma_{0}}($ dev $)=q$ by Lemma 4.4 . On the other hand if $\mathcal{S}_{\sigma_{0}}\left(\operatorname{dev} \circ \widetilde{F}^{-1}\right)=F . q=q=\mathcal{S}_{\sigma_{0}}($ dev $)$ then dev $\circ \widetilde{F}^{-1}=g \circ$ dev for some $g \in \mathrm{PSL}_{2} \mathbb{C}$ by Lemma 4.4, which implies that $F \in \operatorname{Möb}(\sigma)$ again by Lemma 2.7 .

It is natural to ask if this affine action can be reduced to a linear action under a suitable choice of the initial projective structure $\sigma_{0}$. This happens for instance for the Fuchsian uniformization of $X$, as already observed in Lemma 2.17.

Corollary 4.9. Let $\sigma_{0}$ be the Fuchsian uniformization of $X, F \in \operatorname{Aut}(X)$ and $q \in H^{0}\left(X, K^{2}\right)$. Then $F . q=F^{*} q$.

Proof. Since $\sigma_{0}$ is the Fuchsian uniformization we have that $F \in \operatorname{Möb}(\sigma)$ by Lemma 2.17. Therefore $\mathcal{S}_{\sigma_{0}}\left(\widetilde{F}^{-1}\right)=0$ by Lemma 4.4.

On the other hand, as shown by the following corollary, there are plenty of couples $(\sigma, F)$ where $\sigma$ is a projective structure on $X$ and $F$ is a holomorphic automorphism of $X$ which is not projective for $\sigma$.

Corollary 4.10. Let $F \in \operatorname{Aut}(X), F \neq i d_{X}$. If $X$ has genus 2, then also assume $F$ is not the hyperelliptic involution. Let $\sigma_{0} \in \mathcal{P}(X)$ such that $F \in \operatorname{Möb}\left(\sigma_{0}\right)$. Then there exists $\sigma \in \mathcal{P}(X)$ such that $F \notin \operatorname{Möb}(\sigma)$.

Proof. Since $F \in \operatorname{Möb}\left(\sigma_{0}\right)$, we have that $\mathcal{S}_{\sigma_{0}}(F)=0$. In particular the action of $F$ on $H^{0}\left(X, K^{2}\right)$ is the linear action by pullback by Lemma 4.7. The linear action of $\operatorname{Aut}(X)$ by pullback on $H^{0}\left(X, K^{2}\right)$ is known to be faithful by [FK92, p. V.2] if and only if $F$ is not the hyperelliptic involution of a genus 2 surface. Therefore under our hypothesis there exists $q \in H^{0}\left(X, K^{2}\right)$ such that $F . q=F^{*} q \neq q$. By Proposition 4.8 we have that $F$ is not projective for the projective structure $\sigma$ defined by $\mathcal{S}_{\sigma_{0}}(\sigma)=q$.

For instance recall that by picking the Fuchsian uniformization we can satisfy the hypothesis of this statement for any $F \in \operatorname{Aut}(X)$, by Lemma 2.17. We are now ready to discuss the following example.

Example 4.11. Let $X$ be a hyperelliptic Riemann surface, and let $J$ be the hyperelliptic involution. By Proposition 4.8 and Corollary 4.9, the projective structures for which $J$ is a projective diffeomorphism are exactly those that correspond to $J$-invariant holomorphic quadratic differentials with respect to the Fuchsian uniformization of $X$. Notice that when $g=2$ every differential is $J$-invariant, but for $g \geq 3$ the space of $J$-invariant differentials is a proper subspace of $H^{0}\left(X, K^{2}\right)$, so we get a lot of non-trivial examples of non-relatively Hurwitz projective structures. On the other hand by choosing $X$ so that $\operatorname{Aut}(X)=\left\{i d_{X}, J\right\}$ we can obtain non-trivial 
examples of relatively Hurwitz projective structures, namely those corresponding to $J$-invariant differentials with respect to the Fuchsian uniformization; surfaces of this type exist in any genus and were constructed explicitly in [Poo00].

\section{Fuchsian uniformizations and Galois Bely̆ Curves}

In the previous sections we have seen that Fuchsian uniformizations are examples of relatively Hurwitz projective structures (2.17); on the other hand we have provided lots of examples of relatively Hurwitz projective structures which are not related to Fuchsian uniformization (see Examples 2.15 and 4.11). Moreover by Proposition 4.8 relatively Hurwitz projective structures can be seen as fixed points of an affine action of a finite group, so that either there is a unique one, or there is a positive dimensional locus of them. In this section we look for conditions on the underlying Riemann surface under which the Fuchsian uniformization is the unique relatively Hurwitz projective structure on it. As it turns out, such a condition can be conveniently expressed in terms of the behaviour of the quotient by the automorphism group.

Definition 5.1. We say that a Riemann surface $X$ is Galois Belyu if $X / \operatorname{Aut}(X)$ is biholomorphic to $\mathbb{C P}^{1}$ and the quotient map $\pi: X \rightarrow X / \operatorname{Aut}(X)$ is ramified exactly on three points.

The name is motivated by the fact that the quotient map $\pi: X \rightarrow X / \operatorname{Aut}(X)$ is both a Belyu function on $X$ and a (branched) Galois cover. Recall that a Belyı function on a Riemann surface is a non-constant meromorphic function ramified over at most three points. Riemann surfaces admitting such a function are called Belyı̆ curves, and are known to be exactly those surfaces which can be defined over $\overline{\mathbb{Q}}$ as algebraic curves.

The above condition has several classical equivalents, which we list here for the reader's convenience. Recall that a $(a, b, c)$-hyperbolic triangle group $\Delta(a, b, c)$ is the orientation-preserving index-two subgroup of the group of isometries $\bar{\Delta}(a, b, c)$ of the hyperbolic plane $\mathbb{H}^{2}$ which is generated by reflections in the sides of a triangle with angles $\frac{\pi}{a}, \frac{\pi}{b}, \frac{\pi}{c}$.

Theorem 5.2 (Wolfart [Wol97]). Let X be a Riemann surface. Then the following are equivalent:

(i) $X$ is Galois Bely .

(ii) $X \cong \mathbb{H}^{2} / \Gamma$, where $\Gamma$ is a cocompact torsion-free Fuchsian group whose normalizer in $\mathrm{PSL}_{2} \mathbb{R}$ is a hyperbolic triangle group $\Delta$.

(iii) $X$ is an isolated local maximum for the function $Y \mapsto \# \operatorname{Aut}(Y)$.

Remark 5.3. Such surfaces are also known as quasiplatonic surfaces (terminology introduced by Singerman in [Sin01]). We refer to [JW16, Chapter 5] for more details and background about their theory.

The following result allows us to add an item to the previous list in Theorem 5.2 .

Theorem 5.4. Let $X$ be a Riemann surface. Then the following are equivalent:

(i) $X$ is Galois Belyॅ .

(ii) The Fuchsian uniformization of $X$ is the unique relatively Hurwitz projective structure in $\mathcal{P}(X)$. 
Proof. Let us consider the Schwarzian parametrization of $\mathcal{P}(X)$ with respect to the Fuchsian uniformization $\sigma$, so that the action of $\operatorname{Aut}(X)$ is linear on $H^{0}\left(X, K^{2}\right)$ and the zero differential corresponds to $\sigma$. By Proposition 4.8 and Corollary 4.9 a projective structure in $\mathcal{P}(X)$ is relatively Hurwitz if and only if the corresponding holomorphic quadratic differential is invariant by this action of $\operatorname{Aut}(X)$. Invariant holomorphic quadratic differentials constitute a linear subspace of $H^{0}\left(X, K^{2}\right)$, whose dimension can be computed according to the following formula (see [FK92, p. V.2.2])

$$
\operatorname{dim}\left(H^{0}\left(X, K^{2}\right)^{\operatorname{Aut}(X)}\right)=3 g_{0}-3+n
$$

where $g_{0}$ is the genus of $X / \operatorname{Aut}(X)$ and $n$ is the number of critical values of the quotient map $X \rightarrow X / \operatorname{Aut}(X)$. Therefore we see that the zero differential is the unique invariant differential exactly when $X / \operatorname{Aut}(X)$ has genus 0 and the quotient map is ramified exactly over three points (since we always assume the genus of $X$ to be at least 2 , the case $g_{0}=1, n=0$ is not allowed).

Remark 5.5. To get an intuition about the dimension computation in the previous proof, it is worth noticing that, from a Teichmüller-theoretic point of view, the Schwarzian parametrization provides an identification between $\mathcal{P}(X)$ and the cotangent space to Teichmüller space at $X$. The space of relatively Hurwitz projective structures corresponds to the cotangent space at $X$ to the locus of fixed points for the action of $\operatorname{Aut}(X)$ on Teichmüller space, which is a copy of the deformation space for the quotient orbifold $X / \operatorname{Aut}(X)$.

By the above theorem this means that complex projective structures which have strictly more projective automorphisms than any proper deformations are precisely Fuchsian uniformizations of Galois Belyı̆ curves. In particular we obtain the following.

Corollary 5.6. Let $S$ be a surface and $\sigma$ be a projective structure on it. Then $\sigma$ is a Hurwitz projective structure if and only if $\sigma$ is the Fuchsian uniformization for a Hurwitz Riemann surface structure $X$ on $S$.

Proof. Fuchsian uniformizations of Hurwitz Riemann surfaces are Hurwitz projective by Lemma 2.10. Conversely let $\sigma$ be a Hurwitz projective structure; in particular the underlying Riemann surface $X$ is a Hurwitz Riemann surface and $\sigma$ is a relatively Hurwitz projective structure on it. Since Hurwitz Riemann surfaces are Galois Bely $\breve{1}$ (for instance by Theorem 5.2), we have that Theorem 5.4 applies and implies that $\sigma$ must be the Fuchsian uniformization of $X$.

After establishing such a result, it is natural to ask how often one encounters one of the structures covered by Theorem 5.4 and if something special can be said about its group of projective automorphisms (as done in [SPWS17] for the case of translation surfaces). Sporadic and well-studied examples are given by Hurwitz surfaces and Fermat curves $F_{n}=\left\{x^{n}+y^{n}=z^{n}\right\} \subset \mathbb{C P}^{2}$; to obtain examples in any genus $g \geq 2$ one can consider the hyperelliptic curve defined by the equation $y^{2}+x^{m}=1$ in $\mathbb{C}^{2}$ for all values of $m \in \mathbb{N}$. On the other hand only finitely many Galois Bely̌ curves exist for any fixed genus (see [Pop72] and [SPW06]; also see [JW16, Chapter 5] for more details and examples). As far as the groups are concerned, Hurwitz groups (i.e. groups arising as the group of automorphisms of a Hurwitz Riemann surface) are well-known to be quotients of the $(2,3,7)$-triangle 
group (see [Con90] and [Con10]). More generally, as said above in Theorem 5.2, groups arising as the group of automorphisms of a Galois Belyı curve is a quotient of some $(a, b, c)$-hyperbolic triangle group, i.e. they have a presentation of the form $\left\langle x, y \mid x^{a}, y^{b},(x y)^{c}, R\right\rangle$ for some $a, b, c \in \mathbb{N}$ such that $\frac{1}{a}+\frac{1}{b}+\frac{1}{c}<1$ and for some extra relation $R$.

\section{REFERENCES}

[AS60] L. V. Ahlfors and L. Sario. Riemann surfaces. Princeton Mathematical Series, No. 26. Princeton University Press, Princeton, N.J., 1960, pp. $x i+382$.

[Con10] M. Conder. "An update on Hurwitz groups". In: Groups Complex. Cryptol. 2.1 (2010), pp. 35-49.

[Con90] M. Conder. "Hurwitz groups: a brief survey". In: Bull. Amer. Math. Soc. (N.S.) 23.2 (1990), pp. 359-370.

[CV19] P. L. Clark and J. Voight. "Algebraic curves uniformized by congruence subgroups of triangle groups". In: Trans. Amer. Math. Soc. 371.1 (2019), pp. 33-82.

[Dum09] D. Dumas. "Complex projective structures". In: Handbook of Teichmüller theory. Vol. II. Vol. 13. IRMA Lect. Math. Theor. Phys. Eur. Math. Soc., Zürich, 2009, pp. 455-508.

[FK92] H. M. Farkas and I. Kra. Riemann surfaces. Second. Vol. 71. Graduate Texts in Mathematics. Springer-Verlag, New York, 1992, pp. xvi+363.

[GKM00] D. Gallo, M. Kapovich, and A. Marden. "The monodromy groups of Schwarzian equations on closed Riemann surfaces". In: Ann. of Math. (2) $151.2(2000)$, pp. 625-704.

[Gun67] R. C. Gunning. "Special coordinate coverings of Riemann surfaces". In: Math. Ann. 170 (1967), pp. 67-86.

[Hur92] A. Hurwitz. "Ueber algebraische Gebilde mit eindeutigen Transformationen in sich". In: Math. Ann. 41.3 (1892), pp. 403-442.

[JW16] G. A. Jones and J. Wolfart. Dessins d'enfants on Riemann surfaces. Springer Monographs in Mathematics. Springer, Cham, 2016, pp. xiv+259.

[Lev99] S. Levy, ed. The eightfold way. Vol. 35. Mathematical Sciences Research Institute Publications. The beauty of Klein's quartic curve. Cambridge University Press, Cambridge, 1999, pp. x+331.

[LMP09] F. Loray and D. Marín Pérez. "Projective structures and projective bundles over compact Riemann surfaces". In: Astérisque 323 (2009), pp. 223-252.

[Mas69] B. Maskit. "On a class of Kleinian groups". In: Ann. Acad. Sci. Fenn. Ser. A I No. 442 (1969), p. 8.

[Min79] C. D. Minda. "Conformal automorphisms of circular regions". In: Amer. Math. Monthly 86.8 (1979), pp. 684-686.

[Mir95] R. Miranda. Algebraic curves and Riemann surfaces. Vol. 5. Graduate Studies in Mathematics. American Mathematical Society, Providence, RI, 1995, pp. xxii+390.

[Poo00] B. Poonen. "Varieties without extra automorphisms. II. Hyperelliptic curves". In: Math. Res. Lett. 7.1 (2000), pp. 77-82. 
[Pop72] H. Popp. "On a conjecture of H. Rauch on theta constants and Riemann surfaces with many automorphisms". In: J. Reine Angew. Math. 253 (1972), pp. 66-77.

[Sin01] D. Singerman. "Riemann surfaces, Belyi functions and hypermaps". In: Topics on Riemann surfaces and Fuchsian groups (Madrid, 1998). Vol. 287. London Math. Soc. Lecture Note Ser. Cambridge Univ. Press, Cambridge, 2001, pp. 43-68.

[SPW06] J.-C. Schlage-Puchta and J. Wolfart. "How many quasiplatonic surfaces?" In: Arch. Math. (Basel) 86.2 (2006), pp. 129-132.

[SPWS17] J.-C. Schlage-Puchta and G. Weitze-Schmithüsen. "Finite translation surfaces with maximal number of translations". In: Israel J. Math. 217.1 (2017), pp. 1-15.

[Wol97] J. Wolfart. "The "obvious" part of Belyi's theorem and Riemann surfaces with many automorphisms". In: Geometric Galois actions, 1. Vol. 242. London Math. Soc. Lecture Note Ser. Cambridge Univ. Press, Cambridge, 1997, pp. 97-112.

[Zor06] A. Zorich. "Flat surfaces". In: Frontiers in number theory, physics, and geometry. I. Springer, Berlin, 2006, pp. 437-583.

Dipartimento di Matematica - Università di Parma, Parco Area delle Science 53/A, 43132, Parma, Italy

Current address: School of Mathematics - Tata Institute of Fundamental Research, Homi Bhabha Road, Navy Nagar, Colaba, Mumbai 400005, India

E-mail address: gianluca.faraco.math@gmail.com

Dipartimento di Matematica - Università di Bologna, Piazza di Porta San Donato 5, 40126, Bologna, Italy

Current address: Department of Mathematics - Florida State University, 1017 Academic Way, Tallahassee, FL 32306-4510, USA

E-mail address: lorenzo.ruffoni2@gmail.com 